\title{
Association of Alfvén waves and proton cyclotron waves with electrostatic bipolar pulses: magnetic hole events observed by Polar
}

\author{
G. S. Lakhina ${ }^{1}$, B. T. Tsurutani ${ }^{2}$, and J. Pickett ${ }^{3}$ \\ ${ }^{1}$ Indian Institute of Geomagnetism, Colaba, Mumbai, India \\ ${ }^{2}$ Jet Propulsion Laboratory, California Institute of Technology, Pasadena, California, USA \\ ${ }^{3}$ Department of Physics and Astronomy, University of Iowa, Iowa City, IA, USA
}

Received: 29 July 2003 - Accepted: 20 October 2003 - Published: 14 April 2004

Part of Special Issue "International Workshops on Nonlinear Waves and Chaos in Space Plasmas"

\begin{abstract}
Two magnetic hole events observed by Polar on 20 May 1996 when it was in the polar cap/polar cusp boundary layer are studied. Low-frequency waves, consisting of nonlinear Alfvén waves and large amplitude $( \pm 14 \mathrm{nT}$ peakto-peak) obliquely propagating proton cyclotron waves (with frequency $f \sim 0.6$ to $0.7 f_{\text {cp }}$ ), accompanied by electric bipolar pulses (electron holes) and electron heating have been observed located within magnetic holes. It is shown that lowfrequency waves can provide free energy to drive some high frequency instabilities which saturate by trapping electrons, thus, leading to the generation of electron holes.
\end{abstract}

\section{Introduction}

Magnetic holes observed in the vicinity of the magnetopause are characterized by steep local decreases in the magnetospheric magnetic field magnitudes (Cummings and Coleman, 1968; Sugiura et al., 1969). These structures contain enhanced plasma densities maintaining pressure balance (Lühr and Klöcher, 1987; Treumann et al., 1990; Stasiewicz et al., 2001). The magnetic hole-like structures have also been observed on auroral zone magnetic fields, and these have also been called magnetic cavities and magnetic bubbles. Low frequency electromagnetic turbulence often accompanies the magnetic holes. Treumann et al. (1990) have reported that the magnetic holes contain electromagnetic waves with frequencies up to several times the ion cyclotron frequency which may be either whistler waves or ion cyclotron waves.

Stasiewicz et al. (2001) have analyzed waves in the $0-30 \mathrm{~Hz}$ frequency range and have argued that these waves are Doppler-shifted kinetic Alfvén waves (KAWs). They suggested that the magnetic holes are created by a tearing mode reconnection process. Pickett et al. (2002) have analyzed the wave turbulence in the outer cusp boundary layer

Correspondence to: G. S. Lakhina

(lakhina@iig.iigm.res.in) and found that the E/B ratios did not exactly fit the kinetic Alfvén wave (KAW) dispersion curve (contrary to Stasiewicz et al., 2001). They concluded that the waves might be a mixture of dispersive KAWs and perhaps lower hybrid turbulence.

Angelopoulos et al. (2001) have found that the low frequency wave power is consistent with 2-D turbulence with phase velocities smaller than the spacecraft speed and frequency as low as or lower than the oxygen gyrofrequency. They conclude that these waves cannot resonate with and heat the ions.

Recently, Tsurutani et al. (2003) have examined two magnetic hole events in detail using a full complement of Polar field and plasma data on 20 May 1996 when Polar was in the polar cap/polar cusp boundary layer. They found isotropic electron heating, accompanied by nonlinear Alfvén waves, large amplitude $( \pm 14 \mathrm{nT}$ peak-to-peak) obliquely propagating proton cyclotron waves (with frequency $\mathrm{f} \sim 0.6$ to $0.7 \mathrm{f}_{\mathrm{cp}}$ ), and electric bipolar pulses (electron holes), located within magnetic holes/cavities/bubbles. The purpose of this paper is to show that low-frequency waves can provide the free energy needed to drive some high frequency instabilities which saturate by trapping electrons, thus, leading to the generation of electron holes.

\section{Observations}

\subsection{Alfvén waves}

From the analysis of three component magnetic field and plasma data from Polar, Tsurutani et al. (2003) identified two magnetic hole events which occurred on 20 May 1996 during the interval 08:25:17.2 to 08:26:06.4 UT and 08:26:39.8 to $08: 27: 28.9$ UT, respectively. The Polar satellite happens to be in the polar cap/polar cusp boundary layer at these times. The high-time resolution data from the Magnetic Field Experiment (Russell et al., 1995) on Polar are shown in Fig. 1. 
Polar MFE - 20 May 1996 (minimum variance coordinates)

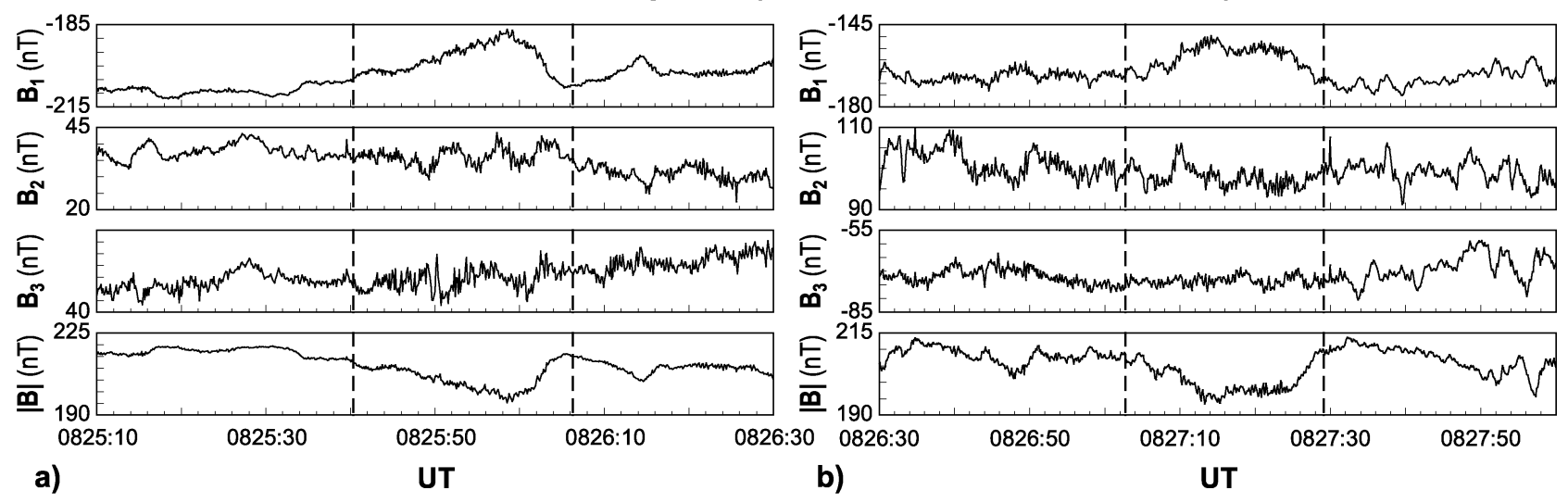

Fig. 1. High time resolution magnetic field data from the Magnetic Field Experiment (MFE) on Polar on 20 May 1996 . The two panels display the magnetic field components around two magnetic hole intervals in minimum variance coordinates. The interval 08:25:17.2 to 08:26:06.4 UT defines the first magnetic hole event. The second magnetic hole occurred in the interval from 08:26:39.8 to 08:27:28.9 UT. In each panel the interval between the two vertical dashed lines were used to define the minimum variance coordinate system (taken from Tsurutani et al., 2003).

The field components are given in a minimum variance coordinate system, where $B_{1}, B_{2}$ and $B_{3}$ are the components along the maximum, intermediate, and minimum variance directions, respectively. Dashed vertical lines include intervals where the minimum variances were calculated. The leftand right-panels correspond respectively to the first and second magnetic hole events. For the first hole event, assuming that this whole interval is one cycle of a wave, the wave in the first magnetic hole is found to be linearly/elliptically polarized $\left(\lambda_{1} / \lambda_{2}=8.4\right)$ and is propagating at a $\sim 79^{\circ}$ angle relative to the ambient magnetic field. There are higher frequency waves superposed on this low frequency wave. For the case of second magnetic hole event, the wave is elliptically polarized $\left(\lambda_{1} / \lambda_{2}=3.9\right)$ and is propagating at $\sim 54^{\circ}$ relative to $\boldsymbol{B}_{\mathbf{0}}$. In the above, $\lambda_{1}$ and $\lambda_{2}$ are the maximum and intermediate eigenvalues of the covariance matrix.

The power spectra of the magnetic field for these magnetic holes showed more or less a steep power law $\left(\mathrm{f}^{-2.3}\right)$ extending from $5 \times 10^{-2} \mathrm{~Hz}$ to $7 \times 10^{-1} \mathrm{~Hz}$ and a noticeable peak at $\sim 2 \mathrm{~Hz}$.

\subsection{Electromagnetic ion cyclotron waves}

Tsurutani et al. (2003) analyzed the output (above $\sim 1 \mathrm{~Hz}$ ) of the low-frequency waveform receiver (LFWR) of the Plasma Wave Instrument (Gurnett et al., 1995) on Polar for the above two magnetic hole events. They found evidence of large amplitude obliquely propagating electromagnetic ion cyclotron waves with a mixture of left- hand and right-hand polarization. Their results corresponding to the time interval of 08:25:57.853 to 08:26:02.493 UT on 20 May 1996 which is inside the first magnetic hole event are shown in Fig. 2. The data are plotted in field-aligned coordinates, where $\hat{z}$ is in the direction of the magnetic field, $\hat{y}$ is in the dawndusk direction orthogonal to $\hat{z}$, and $\hat{x}$ completes the right- hand coordinate system. The top three panels show the electric field components and the lower three panels the magnetic field components for fluctuations above $1 \mathrm{~Hz}$. From the bottom 3 panels, it is clear that there is the presence of large amplitude electromagnetic waves. The transverse magnetic field components are as large as $\sim 14 \mathrm{nT}$ peak-to-peak, and there is a significant parallel component of $\sim 7 \mathrm{nT}$ peak-topeak. The average wave frequency is $2.3 \mathrm{~Hz}$. The local proton cyclotron frequency is $3.05 \mathrm{~Hz}$, so the wave frequency is $0.75 \mathrm{f}_{\mathrm{cp}}$. Figure 2 also indicates the presence of intense electrostatic waves within the interval. At 08:26:02.333 to 08:26:02.493 UT, waves with peak-to-peak amplitudes of $\sim 6 \mathrm{mV} / \mathrm{m}$ are present. These waves have a frequency of $\sim 31 \mathrm{~Hz}$. Because the bandpass for this channel rolls off at $25 \mathrm{~Hz}$ (the $3 \mathrm{~dB}$ point), the true wave amplitudes may be significantly larger.

\subsection{Electrostatic bipolar pulses (electron holes) and high- frequency waves}

Analysis of the data from the PWI high frequency waveform receiver (HFWR) show that the waveform and power spectral density of the waves changes over very short time scales $(\sim 10 \mathrm{~ms}$ or so) inside the magnetic holes. Tsurutani et al. (2003) detected electric bipolar pulse (electron hole) onset inside the magnetic holes during the electron heating events associated with the magnetic holes. The growth rate of the bipolar pulses from onsets to attaining the peak amplitudes were found to be $\sim 0.25 \mathrm{f}_{\mathrm{ce}}$, where $\mathrm{f}_{\mathrm{ce}}$ is the electron cyclotron frequency. The peak-to-peak amplitudes of the $E_{\|}$ signal were found to be $\sim 2$ to $9 \mathrm{mV} / \mathrm{m}$. The $E_{\|}$signals were sometimes accompanied with a transverse $\left(E_{\perp}\right)$ electric component of $\sim 3-4 \mathrm{mV} / \mathrm{m}$. In Figs. 3 and 4 we show the waveform plots obtained from high time resolution HFWR data selected from the first and second magnetic hole events. 


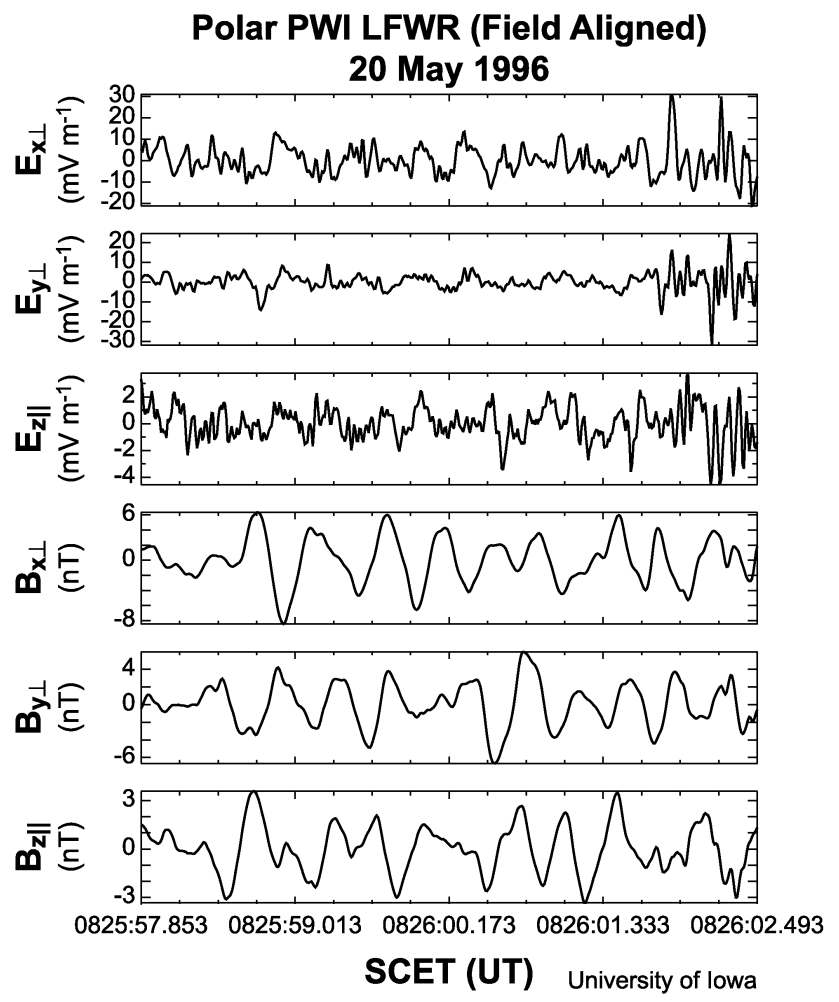

$$
\begin{array}{rlrl}
R_{E} & =6.05 & & \\
L & =27.32 & M L T & =11.62 \\
\lambda_{m} & =61.96 & F_{c p} & =3.05 \mathrm{~Hz}
\end{array}
$$

Fig. 2. Low frequency waves during the first magnetic hole interval. The bandpass is $1 \mathrm{~Hz}$ to $25 \mathrm{~Hz}$. The coordinates are magnetic fieldaligned. Electromagnetic waves with peak-to-peak amplitudes as large as $\sim 14 \mathrm{nT}$ are detected. The waves are a mixture of left-hand and right-hand polarization as detected in the spacecraft frame. The $\sim 2.3 \mathrm{~Hz}$ waves occur at $\sim 0.75 f_{\mathrm{cp}}$ and are thus believed to be proton cyclotron waves (taken from Tsurutani et al., 2003).

In both Figures, the data are plotted in field-aligned coordinates, as described above. The top three panels show the electric field components and the lower three panels the magnetic field components for fluctuations above $20 \mathrm{~Hz}$.

Figure 3 corresponds to the time interval of 08:25:45.145 to $08: 25: 45.202$ UT on 20 May 1996 and is contained within the time period of the first magnetic hole event. The parallel electric field component shows bipolar and off-set bipolar pulses (panel 3) with peak-to-peak amplitudes of $\sim 3-$ $6 \mathrm{mV} / \mathrm{m}$. The transverse electric components (panels 1 and 2 ) have smaller amplitudes and they show modulations with different periods. All the three magnetic components show slow modulations. The slow modulations appears to be near the lower hybrid frequency $(\sim 150-200 \mathrm{~Hz})$, but we cannot be absolutely certain as these are not resolved very well because of their being close to the lower end of the band pass filter. The two perpendicular magnetic components have outof-phase modulations.

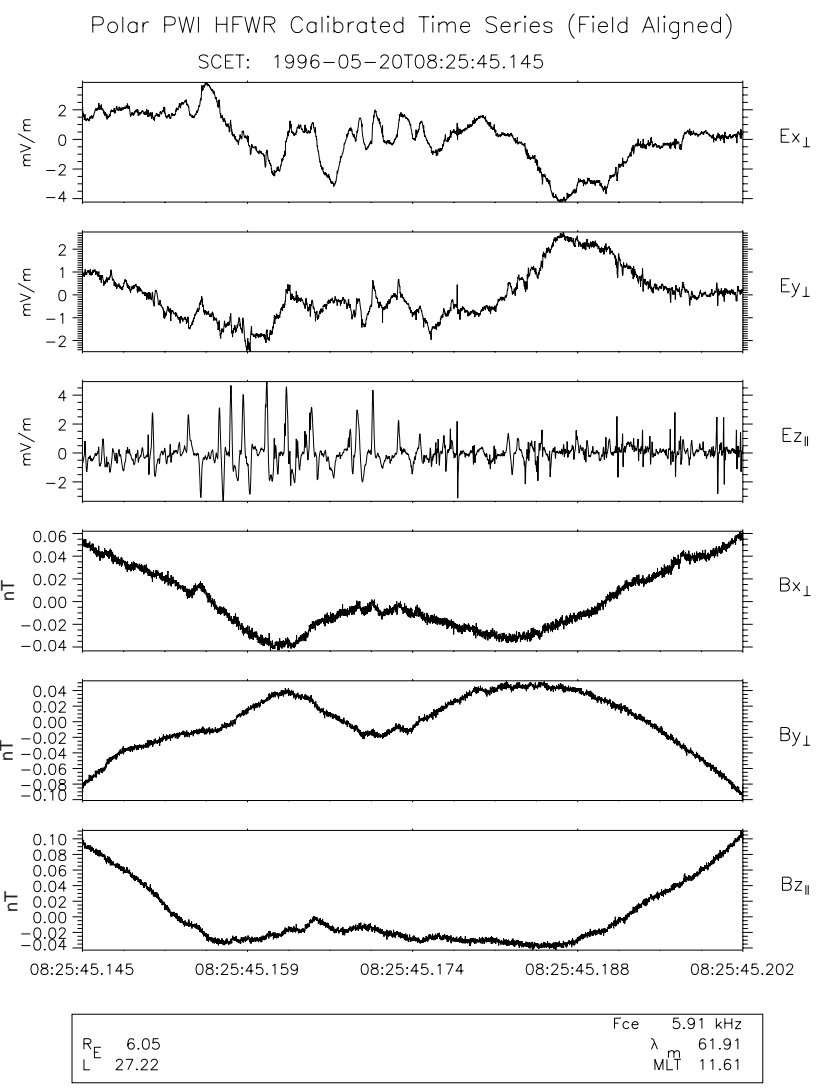

Fig. 3. Shows the waveform plots from high time resolution HFWR data for the interval of 08:25:45.145 to 08:25:45.202 UT. The data illustrate waves present during the first magnetic hole event in fieldaligned coordinates. The top three panels show the electric field components and the lower three panels the magnetic field components for fluctuations above $20 \mathrm{~Hz}$. The parallel electric field component show bipolar and off-set bipolar pulses (panel 3) with peakto-peak amplitudes $\sim 3-6 \mathrm{mV} / \mathrm{m}$.

Figure 4 corresponds to the time interval of 08:27:17.145 to $08: 27: 17.202$ UT on 20 May 1996 and is contained within the time period of the second magnetic hole event. Here all components of electric and magnetic field show slow modulations on which high frequency pulses are superposed. The parallel electric field component shows some bipolar structures with peak-to-peak amplitudes of $\sim 2-3 \mathrm{mV} / \mathrm{m}$ during latter half of the interval.

Figures 5 and 6 show the power spectrum of the electric and magnetic components for the intervals of Figs. 3 and 4, respectively. Figure 5 indicates that the power spectral density decreases with increasing frequency for all components except $E_{\|}$. The $E_{\|}$component does not follow this trend. It increases up to about $1 \mathrm{kHz}$ and then decreases with increasing frequency. The spectrum does not show any wellresolved peaks which could identify the existence of certain plasma modes. There could, however, be a possible peak near $150-200 \mathrm{~Hz}$ (i.e. close to the lower hybrid frequency) as 


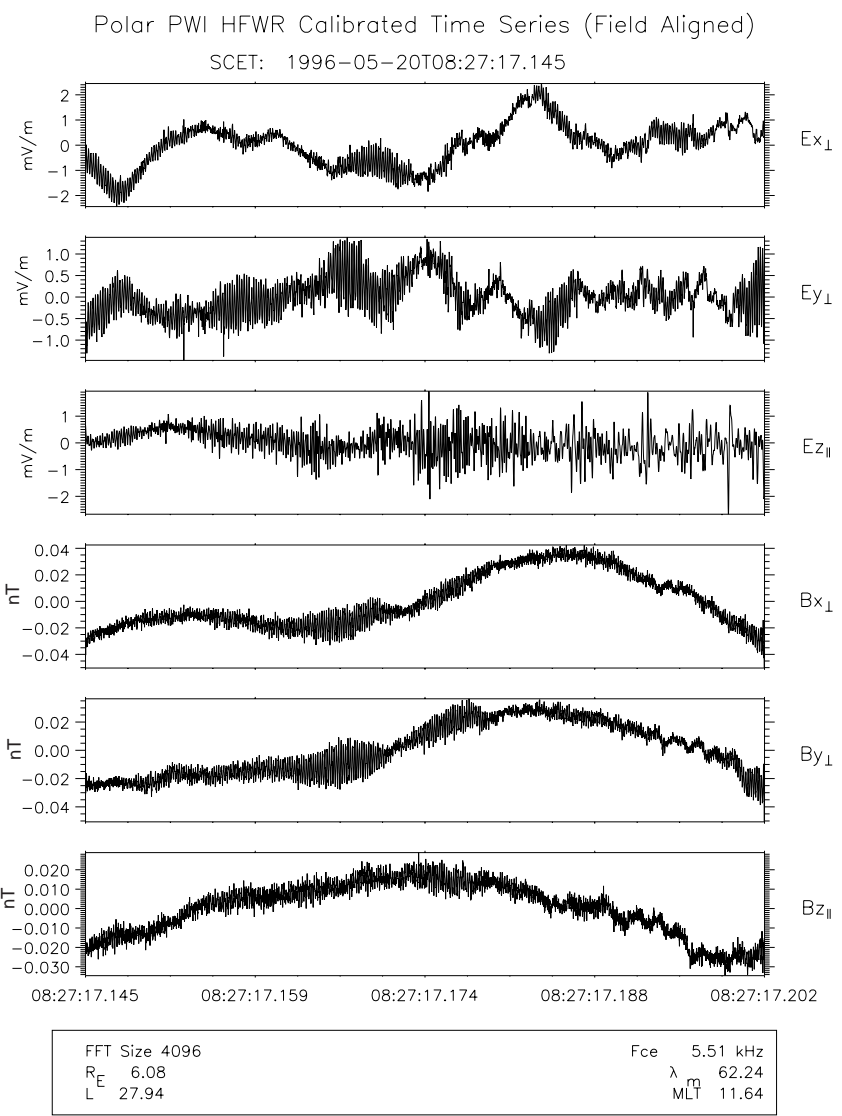

Fig. 4. same format as Fig. 3 but for the time interval of 08:27:17.145 to 08:27:17.202 UT on 20 May 1996 during the second magnetic hole event. Here all components of electric and magnetic field show slow modulations on top of which high frequency pulses are superposed. The parallel electric field component shows some bipolar structures with peak-to-peak amplitudes of $\sim 2-3 \mathrm{mV} / \mathrm{m}$ (see the latter half of the interval).

seen by the higher intensity $E_{\perp}$ component as compared to the $E_{\|}$component, but one cannot be sure as it is close to the lower end of the band pass filter. Figure 6 shows a clear prominent peak near $5.1 \mathrm{kHz}$ which is below electron cyclotron frequency $\mathrm{f}_{\mathrm{ce}} \approx 5.91 \mathrm{kHz}$ in all electric and magnetic components. It is possibly due to the electromagnetic electron cyclotron wave mode which is almost always observed in the turbulent boundary layer (Pickett et al., 2001). It is probable that some of the high frequency modulations noted in Fig. 4 are due to these electromagnetic electron cyclotron waves. The peak near the lower hybrid frequency cannot be resolved here again though $E_{\perp}$ components are more intense than the $E_{\|}$component, similar to Fig. 5. We may point out that electron plasma frequency $\mathrm{f}_{\mathrm{pe}} \sim 28 \mathrm{kHz}$ cannot be seen as it falls outside the highest frequency $(=25 \mathrm{kHz})$ that can be measured with HFWR.

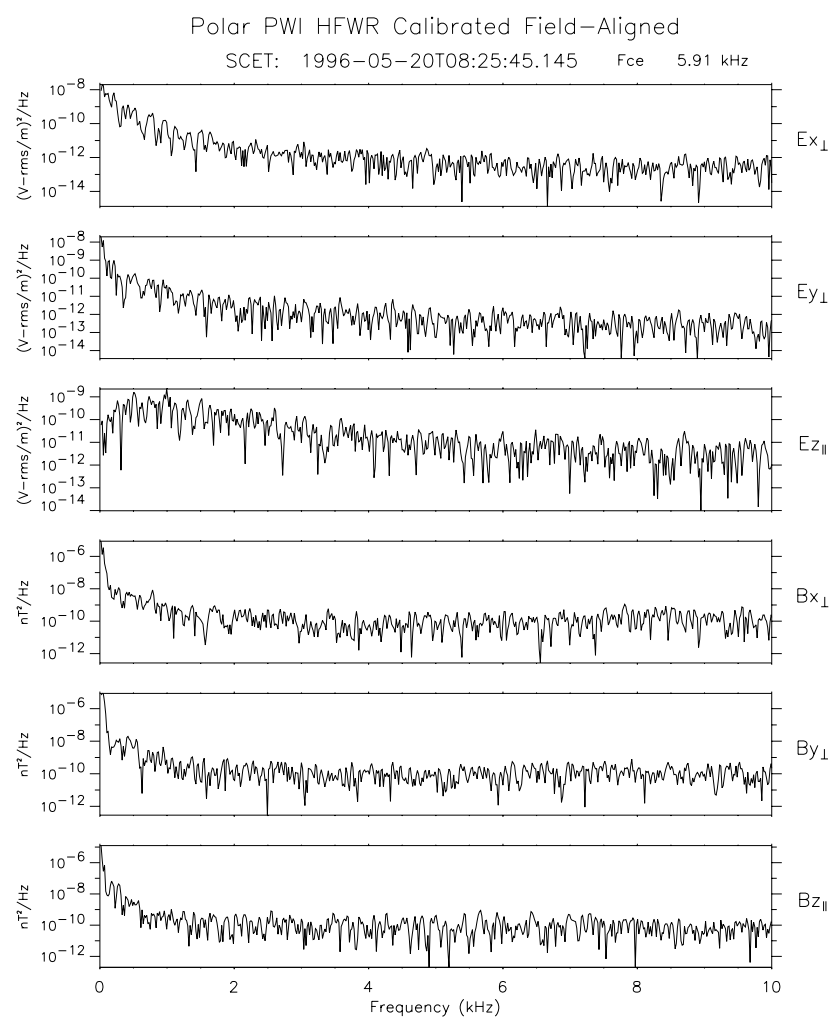

Fig. 5. Shows the power spectrum of the electric and magnetic components for the intervals of Figs. 3 and 4 . Figure 5 indicates that the power spectral density decreases with increasing frequency in all but $E_{\|}$component and does not show any well resolved peaks which could be identified as being caused by some plasma modes. There could be a possible peak near $150-200 \mathrm{~Hz}$ (i.e. close to the lower hybrid frequency) as seen by the higher intensity $E_{\perp}$ panels as opposed to the $E_{\|}$panel, but one cannot be sure as it is close to the lower end of the band pass filter.

\subsection{Electron heating events}

Tsurutani et al. (2003) looked into the plasma data, obtained by the Hydra instrument (Scudder et al., 1995) onboard Polar, during the time of above two magnetic hole events. They found evidence of electron heating occurring simultaneously with electron and ion density increases. The electrons are heated to $\sim 100-200 \mathrm{eV}$. The electron distributions during the background interval and during the electron heating interval were found to be isotropic. They did not find any evidence of electron bi-directional streaming in either case. However, they notice a field-aligned ion beam during the background interval. The electron and ion density increases were observed inside the magnetic holes.

\section{Generation of high-frequency waves and electron holes}

The most popular mechanism for the generation of electron holes is based on electron beam instabilities as suggested by 
Omura et al. (1994,1996), Kojima et al. (1997) and Miyaki et al. (1998) for explaining Geotail observations of bipolar solitary pulses in the plasma sheet boundary layer (Matsumoto et al., 1994). In this mechanism, the nonlinear saturation of electron beam instabilities occurs by electron trapping in the large amplitude waves leading to the formation of isolated Bernstein-Green-Kriskal (BGK) potential structures which reproduce well the signature of electron holes. The electric bipolar structures observed by FAST (Ergun et al., 1998a, b) and Polar (Franz et al., 1998; Tsurutani et al., 1998, 2001; see Lakhina et al., 2000, for a review) have been interpreted in terms of BGK (Bernstein et al., 1957) phase space electron holes (Muschietti et al., 1999a, b). Goldman et al. (1999a, b) and Singh et al. (2001a, b) have explained the bipolar structures in terms of nonlinear two-stream electron instabilities. For the case of magnetic hole events analyzed here, there is no indication of long-lasting bi-directional electron beams. This would preclude the possibility of electron hole generation by electron beams. In view of this, Tsurutani et al. (2003) suggested the possibility that the parallel electric field component of the obliquely propagating proton cyclotron waves can provide a mechanism for short duration bi-directional electron beams which could provide free energy for the fast growing electron beam instabilities. Here we propose that nonlinear Alfvén waves and ion cyclotron waves can supply free energy to excite high frequency wave modes that eventually lead to electron holes.

\subsection{Excitation of high-frequency instabilities by} low-frequency waves

Khazanov et al. (1996, 2000) have demonstrated that largeamplitude low-frequency waves can generate lower hybrid waves in the auroral zone and ring current region. The free energy for driving the lower hybrid waves comes from the perpendicular current arising from different polarization drifts between electrons and ions. To understand this mechanism let us discuss first the motion of charged particles in the presence of low-frequency waves.

In the presence of low-frequency waves (e.g. Alfvén wave) propagating obliquely to the ambient magnetic field $\boldsymbol{B}_{\mathbf{0}}$ (taken to be along $z$-direction), the electrons and ions will acquire different velocities $\boldsymbol{U}_{\boldsymbol{e}}$, and $\boldsymbol{U}_{\boldsymbol{i}}$. If the frequency of the low-frequency wave $\omega_{0}$ is much lower than the electron cyclotron frequency $\omega_{c e}$, the electron and ion velocities are given by (Khaznov et al., 1996),

$$
\begin{aligned}
& \boldsymbol{U}_{\boldsymbol{e}}=\frac{c \boldsymbol{E} \times \boldsymbol{B}_{\mathbf{0}}}{B_{0}^{2}}-i \frac{e(\boldsymbol{E} \cdot \boldsymbol{b}) \boldsymbol{b}}{\omega_{0} m_{e}} \\
& \boldsymbol{U}_{\boldsymbol{i}}=\frac{e}{m_{i}\left(\omega_{c i}^{2}-\omega_{0}^{2}\right)}\left[\omega_{c i}(\boldsymbol{E} \times \boldsymbol{b})+i \omega_{0}(\boldsymbol{E} \times \boldsymbol{b}) \times \boldsymbol{b}\right] \\
&+i \frac{e(\boldsymbol{E} \cdot \boldsymbol{b}) \boldsymbol{b}}{\omega_{0} m_{i}}
\end{aligned}
$$

where $\boldsymbol{E}=\boldsymbol{E}_{\mathbf{0}} \exp \left(-i \omega_{0} t\right)$ is the electric field of the lowfrequency wave, $\omega_{c i}$ is the ion cyclotron frequency, and $\boldsymbol{b}$ is unit vector along $\boldsymbol{B}_{\mathbf{0}}$. It is clear from Eqs. (1) and (2) that

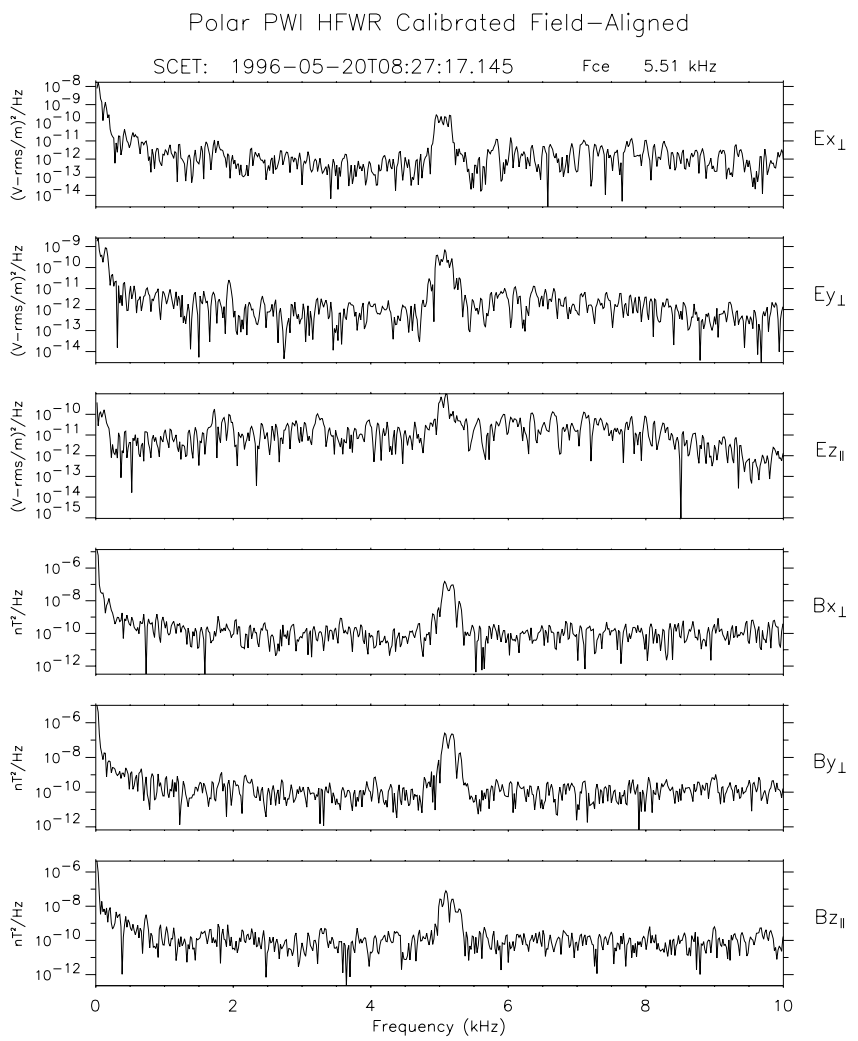

Fig. 6. Shows the power spectrum of the electric and magnetic components for the interval of Fig. 4. There is a clear and prominent peak at a frequency below the electron cyclotron frequency $f_{c e} \approx 5.91 \mathrm{kHz}$. This is present in all electric and magnetic field components.

electrons and ions would have relative velocity $\boldsymbol{U}=\left(\boldsymbol{U}_{\boldsymbol{e}}-\boldsymbol{U}_{\boldsymbol{i}}\right)$ with components in both the parallel and perpendicular directions to $\boldsymbol{B}_{\mathbf{0}}$. For $\boldsymbol{E}_{\perp \mathbf{0}}=E_{x 0} \boldsymbol{x}$, these are given by,

$$
\begin{aligned}
& \operatorname{Re} U_{\|}=\frac{e E_{z 0}}{\omega_{0} m_{e}} \sin \omega_{0} t \\
& \operatorname{Re} U_{x}=-\frac{\omega_{0} E_{x 0}}{\omega_{c i}^{2} m_{e}} \sin \omega_{0} t \\
& \operatorname{Re} U_{y}=\frac{c E_{x 0}}{B_{0}} \cos \omega_{0} t-\frac{e \omega_{c i} E_{x 0} \cos \omega_{0} t}{m_{i}\left(\omega_{c i}^{2}-\omega_{0}^{2}\right)}
\end{aligned}
$$

Case A: Low-frequency waves without a parallel electric field component

For the case of low-frequency $\left(\omega_{0} \ll \omega_{c i}\right)$ Alfvén waves with no parallel electric field $\left(E_{\|}=0\right)$, it is seen from Eqs. (3)-(5) that the perpendicular current $U_{x}$, arising from the polarization drift, can drive the lower hybrid wave instability (Khazanov et al., 1996; 2000) provided

$$
\frac{E_{x 0}^{2}}{8 \pi n T} \geq \frac{\omega_{c i}^{4}}{\omega_{0}^{2} \omega_{p i}^{2}}
$$


where $n$ and $T$ are the ion density and temperature (the electron temperature is assumed smaller that the ion temperature, i.e. $T_{e} \ll T_{i}$ ) and $\omega_{p i}$ is the ion plasma frequency. Considering the typical plasma and field parameters for magnetic holes as $n=10 \mathrm{~cm}^{-3}, B_{0}=200 \mathrm{nT}, T_{e}=(100-200) \mathrm{eV}, T_{i}=1000 \mathrm{eV}$, and taking $\omega_{0} / \omega_{c i}=0.1$ (i.e. $f_{0}=\omega_{0} / 2 \pi \approx 0.3 \mathrm{~Hz}$ ), the minimum electric field required for instability is about $E_{x 0} \approx 870 \mathrm{mV} / \mathrm{m}$.

Case B: Ion cyclotron waves with $E_{\|}=0$

For the case of $\omega_{0} \sim \omega_{c i}$ and $E_{\|}=0$, it is seen from Eqs. (3)(5), that the perpendicular current due to $U_{y}$ (Hall current) can drive several instabilities, such as lower hybrid, ion acoustic wave, etc., depending upon the ratio of $U_{y} / V_{t i}$, where $V_{t i}$ is the ion thermal speed. The minimum electric field required corresponds to $U_{y}>V_{t i}$, and is given by the inequality,

$E_{x 0}>\frac{V_{t i} B_{0}}{c}\left[\frac{\omega_{c i}^{2}}{\omega_{0}^{2}}-1\right]$.

Again considering the typical plasma and field parameters for the magnetic hole events observed by Polar, and taking $\omega_{0} / \omega_{c i} \approx 0.6$, the threshold electric field for exciting instability would be $E_{x 0} \approx 1.0 \mathrm{~V} / \mathrm{m}$.

Case C: Low-frequency waves with finite parallel electric field $\left(E_{\|} \neq 0\right)$ component

The obliquely propagating Alfvén waves or ion cyclotron waves can have a substantial parallel electric field component. The finite $E_{\|}$component could give rise to strong electric currents carried mainly by the electrons propagating parallel to the ambient magnetic field. This current can drive several high-frequency electron-beam type instabilities.

The minimum electric field required for the bi-stream electron instability is given by the inequality (Omura et al., 1996),

$\frac{e E_{z 0}}{\omega_{0} m_{e}}>V_{t e}$,

where $V_{t e}$ is the electron thermal speed. This instability gets saturated by trapping electrons and could lead to electron holes.

Similarly, the parallel current can give rise to lowerhybrid, ion acoustic and electrostatic ion cyclotron type instabilities provide the following inequalities are satisfied,

$V_{t i}<\frac{e E_{z 0}}{\omega_{0} m_{e}}<V_{t e}$.

Considering $T_{e}=200 \mathrm{eV}$ and $\omega_{0} / 2 \pi \approx 0.3 \mathrm{~Hz}$, the minimum parallel electric field satisfying the inequality is about $E_{\|}=0.06 \mathrm{mV} / \mathrm{m}$. Since the $E_{\|}$electric components associated with the obliquely propagating ion cyclotron waves (or Alfvén waves) could be $\sim 1 \mathrm{mV} / \mathrm{m}$, they are expected to excite several electron-beam type instabilities. In the next section we consider a simplified model of high frequency instabilities excited by the counter-streaming electron beams.

\section{Two-Stream instabilities in a magnetized plasma}

Let us consider an electron-proton plasma containing lowfrequency waves which are propagating obliquely with respect to the ambient uniform magnetic field $\boldsymbol{B}_{\mathbf{0}}=B_{0} \boldsymbol{z}$. We consider two counterstreaming electron beams propagating along the magnetic field. For simplicity we assume that these counterstreaming electron beams arise from acceleration by the parallel electric field components of the obliquely propagating ion cyclotron waves, as suggested by Tsurutani et al. (2003). For simplicity, we would consider here the excitation of electrostatic waves by the counterstreaming electron beam only and leave the generation of electromagnetic waves for a future study. The dispersion relation for the electrostatic waves can be written (Singh et al., 2001b) as,

$k_{\perp}^{2} \varepsilon_{\perp}+k_{\|}^{2} \varepsilon_{\|}=0$,

where

$$
\begin{gathered}
\varepsilon_{\perp}=1-\frac{\omega_{p 1}^{2}}{\left(\omega-k_{\|} V_{b 1}\right)^{2}-\omega_{c e}^{2}} \\
-\frac{\omega_{p 2}^{2}}{\left(\omega-k_{\|} V_{b 2}\right)^{2}-\omega_{c e}^{2}}, \\
\varepsilon_{\|}=1-\frac{\omega_{p 1}^{2}}{\left(\omega-k_{\|} V_{b 1}\right)^{2}}-\frac{\omega_{p 2}^{2}}{\left(\omega-k_{\|} V_{b 2}\right)^{2}},
\end{gathered}
$$

and $k_{\perp}$ and $k_{\|}$are the components of the wave vector $\boldsymbol{k}$ along perpendicular and parallel directions to the ambient magnetic field. Here $\omega_{p 1}\left(\omega_{p 2}\right)$ and $V_{b 1}\left(V_{b 2}\right)$ the electron plasma frequency and the drift speed of the electron beams 1 and 2, respectively.

We have solved the dispersion relation (10) numerically by Mathematica for the parametric range relevant to the observed low-frequency waves and plasma during the magnetic hole events by Polar. The results are shown in Figs. 7-9. In all of these figures, the real frequency, $\omega_{r}$, and the growth rate, $\gamma$, are normalized with respect to $\omega_{p 0}$. Here $\omega_{p 0}$ is the electron plasma frequency assuming the total electron density $n_{0}=n_{1}+n_{2}$, where $n_{1}\left(n_{2}\right)$ is the density of the electron beam 1(2). The wave number $k$ is normalized with respect to $\lambda_{D 1}=V_{t 1} / \omega_{p 0}$, the Debye length defined by assuming the temperature of beam 1 and taking the total electron density.

Figure 7 shows the variation of normalized real frequency (panel a) and growth rate (panel b) against $k \lambda_{D 1}$ for fractional density $R=n_{2} / n_{0}=0.1,0.3$ and 0.5 . It is seen that real frequencies decrease as $R$ increases (cf. curves 1, 2 and 3 of panel a). The growth rates increases with an increase of $R$ from 0.1 to 0.5 (cf. curves 1,2 and 3 of panel b). With further increase in $R$, the growth rate starts decreasing and instability vanishes for $R=1.0$ (not shown). The range of unstable wave numbers increases when $R$ is increased.

Figure 8 shows the dispersion relation for different values of the wave angle of propagation, i.e. $\tan \theta=k_{\perp} / k_{\|}=0.0,1.0$ and 5.0 for the curves 1,2 and 3 respectively. It is seen that at $\tan \theta=1$ and 5 , there are 3 unstable regions in wave number space instead of just one unstable region for the $\tan \theta=0$ 

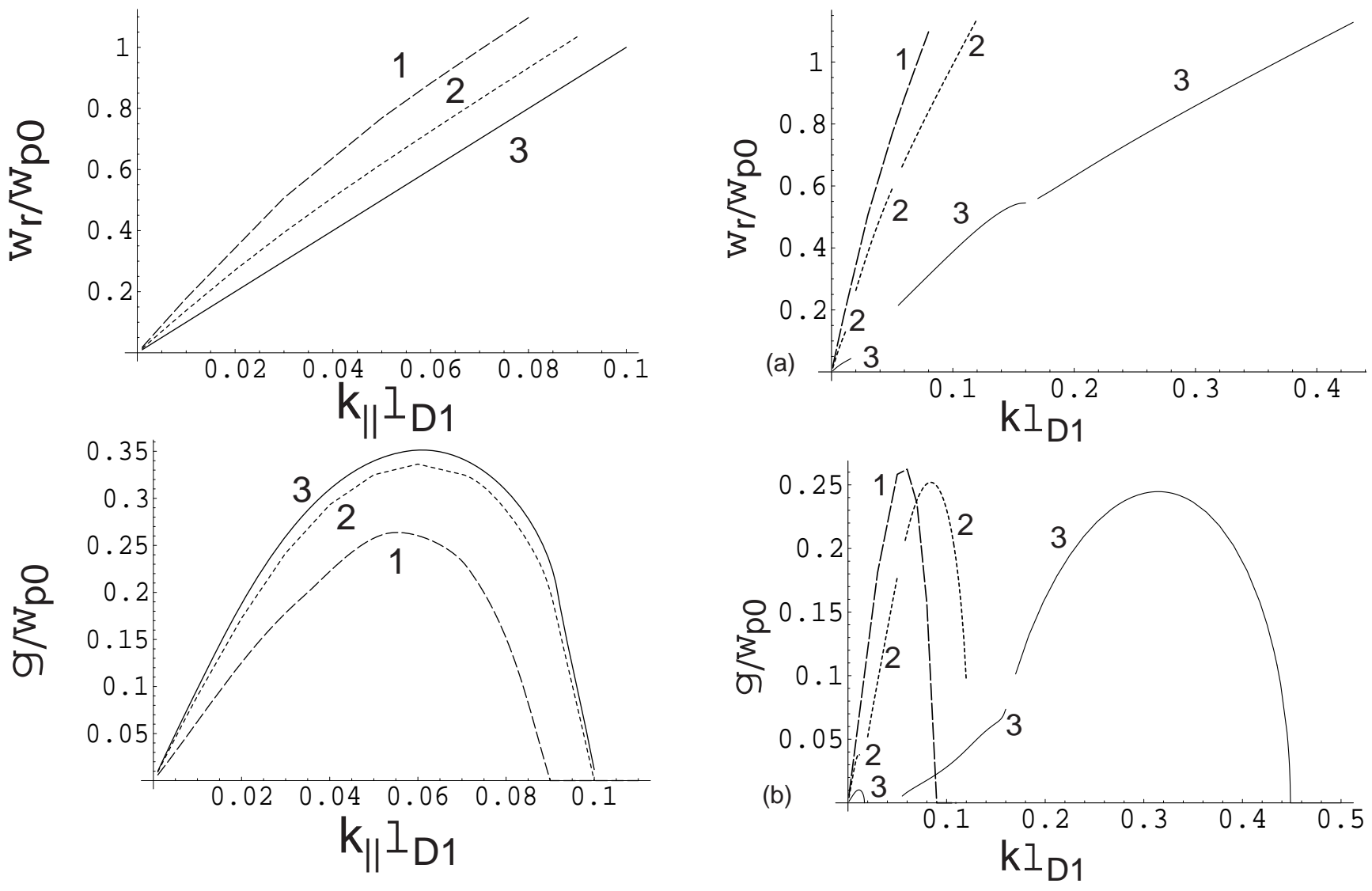

Fig. 7. The variation of normalized real frequency $\omega_{r} / \omega_{p 0}$ (panel (a)) and growth rate $\gamma / \omega_{p 0}$ (panel (b)) plotted against $k \lambda_{D 1}$ for fractional density values $R=n_{2} / n_{0}=0.1,0.3$ and 0.5 for the curves 1,2 and 3 , respectively. Other parameters are: $V_{b 1}=0.0, V_{b 2}=20.0, T_{b 1} / T_{b 2}=1.0$, and the electron cyclotron to electron plasma frequency ratio, $\omega_{c e} / \omega_{p 0}=0.2$.

case. It appears that the unstable region corresponding to the highest values of $k \lambda_{D 1}$ is the Langmuir beam-plasma mode and that corresponding to the lowest values is the ion acoustic/lower hybrid type. The middle unstable region covers the intermediate frequency range of $\omega_{l h}<\omega_{c e}<\omega_{p 0}$. The unstable wave numbers shift towards higher values of $k \lambda_{D 1}$ (i.e. to shorter wavelengths) for large propagation angles. However the growth rates tend to decrease as $\tan \theta$ increases.

Figure 9 shows the dispersion relations for different values of the ratio $\omega_{c e} / \omega_{p 0}(0.5,1.0$ and 5.0) for the curves 1 , 2 and 3 , respectively. Here again there are more than one unstable wave number regimes for some parameters. However, it is interesting to note that the number of unstable windows decreases as $\omega_{c e} / \omega_{p 0}$ increases. The growth rates corresponding to the first unstable regime show increase with an increase of the $\omega_{c e} / \omega_{p 0}$ ratio.

Now, for the parameters of the magnetic hole events, we can take $n_{0}=10 \mathrm{~cm}^{-3}, \quad B_{0}=200 \mathrm{nT}, \quad T_{e}=200 \mathrm{eV}$ and $T_{i}=1000 \mathrm{eV}$, this gives $f_{p 0}=\omega_{p 0} / 2 \pi=28.4 \mathrm{kHz}$,

$f_{c e}=\omega_{c e} / 2 \pi=5.6 \mathrm{kHz}$, and $\lambda_{D 1}=30 \mathrm{~m}$. Also, we get $\omega_{c e} / \omega_{p 0}=0.197$ which is quite close to the value 0.2 taken for Figs. 7-9. Further, our choice of normalized relative drift speed, $U=\left(V_{b 2}-V_{b 1}\right) / V_{t 1}=20$ appears to be reasonable in view of the fact that the observed parallel electric fields associated with the obliquely propagating low-frequency waves are at least one order of magnitude higher than the threshold value. Then, from Figs. 7-9, we observe that real frequencies of the excited modes are $f \sim(0.01-1.2) \quad f_{p 0}=(0.28-34) \mathrm{kHz}$. The peak growth rate $\gamma$ varies over a wide range, from $\sim(0.01-0.35) \quad f_{p 0}=(0.28-9.9) \mathrm{kHz}$. The unstable wave numbers associated with the maximum growth rates occur in the range of $k \lambda_{D 1} \sim(0.05-0.5)$. This would give the corresponding unstable wavelengths $\lambda=\left(2 \pi / k \lambda_{D 1}\right)$. $\lambda_{D 1}=(375-3750) \mathrm{m}$. The e-folding time of the excited modes is $\sim(0.1-3) \mathrm{ms}$, so we expect that it would take a few tens of ms to saturate the instabilities. Simulations of Omura et al. $(1994,1996)$ show that the bistream instability starts 


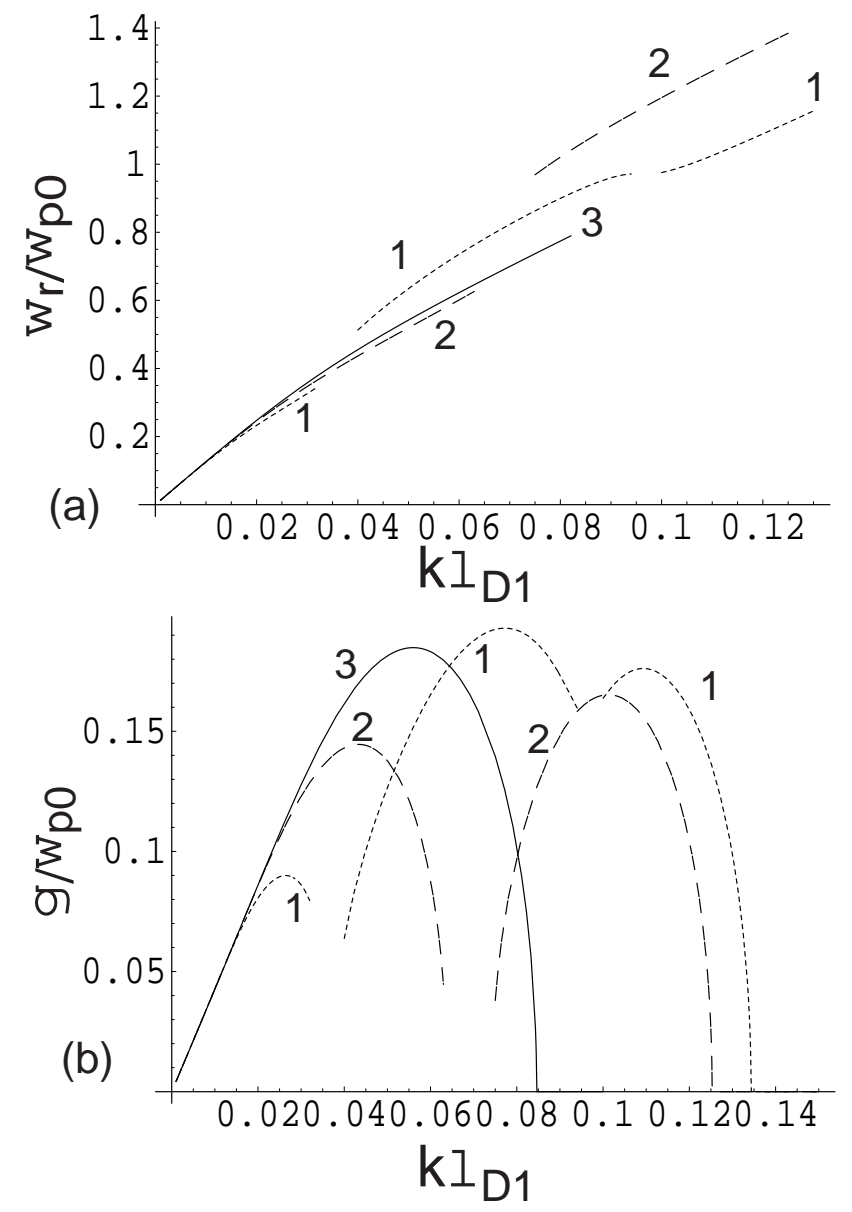

Fig. 9. The variation of normalized real frequency $\omega_{r} / \omega_{p 0}$ (panel (a)) and growth rate $\gamma / \omega_{p 0}\left(\right.$ panel (b)) plotted against $k \lambda \lambda_{D 1}$ for $R=n_{2} / n_{0}=0.1, \quad \tan \theta=k_{\perp} / k_{\|}=1.0$, and $\omega_{c e} / \omega_{p 0}=0.5, \quad 1.0$ and 5.0 for the curves 1,2 and 3 , respectively. Other parameters are the same as in Fig. 7.

trapping the electrons after time steps of $t=(100-400) t_{p 0}$, where $t_{p 0}=1 / f_{p 0}$ is the time period of the electron plasma frequency. Further, particle simulations of beam-plasma instabilities by Singh et al. (2001) also show that electron holes usually occur for time period of (100-400) $t_{p 0}$. For the case of electron holes in the present case, it would translate to time scale of $\sim(3-15) \mathrm{ms}$. Therefore we expect that some of the beam modes studied here would saturate by trapping electrons and would produce signatures of bipolar pulses or electron holes. This time scale is short enough compared to the time period $(\sim 0.5 \mathrm{~s}$ for the cyclotron waves and a few tens of seconds for the Alfvén waves) of the low-frequency waves such that the assumption of constant electric field acting on the plasma is justified.

\section{Discussion}

Tsurutani et al. (2003) put forward a scenario to explain the observations of low-frequency waves (Alfvén and electromagnetic ion cyclotron waves) in conjunction with electron heating and electric bipolar pulses. According to them, the low-frequency waves are the free energy source for the electron heating and electric bipolar pulses. We have extended their argument and tried to provide a theoretical model for this scenario based on the idea first proposed by Khazanov et al. $(1996,2000)$ for the generation of lower hybrid waves by large amplitude low-frequency waves in the auroral zone and ring current region. Our analysis shows that the instabilities driven by the perpendicular current are not possible for the case of magnetic hole events as they require very large low-frequency wave amplitude $E_{\perp 0} \sim 1 \mathrm{~V} / \mathrm{m}$. On the other hand, the observed $E_{\|}$of the obliquely propagating low-frequency wave (Alfvén and electromagnetic ion cyclotron waves) are strong enough to give rise to several high-frequency electron beam instabilities. It is hypothesized that some of these modes could saturate by trapping electrons leading to electron hole generation. We have also shown the presence of clear electromagnetic electron cyclotron mode from the analysis of HFWR data. The present model is electrostatic and cannot explain this mode. This will be investigated and reported elsewhere.

The electron heating events are most likely produced either by the Ponderomotive force of the low-frequency waves or by the heating/acceleration by the parallel electric field component of the obliquely propagating ion cyclotron waves as suggested by Tsurutani et al. (2003). Some of the high frequency modes could also produce electron heating along the magnetic field direction. We have not addressed the issue related to the generation of low-frequency waves. One possible source of the Alfvén waves is creation by the pulsed magnetic reconnection in the cusp with consequential propagation into the magnetosphere to the spacecraft location. Furthermore, we have also not addressed the issue of generation of electromagnetic electron cyclotron waves. These two fascinating topics are left for the future studies.

Acknowledgements. GSL would like to thank Y. Kamide for the warm hospitality during his stay at Solar-Terrestrial Environment Laboraroty, Nagoya University, Toyokawa, Japan. Portions of this research were performed at the Jet Propulsion Laboratory, California Institute of Technology, under contract with the National Aeronautics and Space Administration. The research conducted at the University of Iowa was under NASA/GSFC Grants NAG57943 and NAG5-9561.

Edited by: J. Büchner

Reviewed by: one referee

\section{References}

Angelopoulos, V., Mozer, F. S., Bonnell, J., Temerin, M., Somoza, M., Peterson, W. K., Collin, H. L., and Giles, B.: Wave power studies of cusp crossings with the Polar satellite, J. Geophys. Res., 106, 5987-6006, 2001.

Bernstein, I. B., Greene, J. M., and Kruskal, M. D.: Exact nonlinear plasma oscillations, Phys. Rev. Lett., 108, 546-550, 1957. 
Cummings, W. D. and Coleman Jr. P. J., : Magnetic fields in the magnetopause and vicinity at synchronous altitude, J. Geophys. Res., 73, 5699, 1968.

Ergun, R. E., Carlson, C. W., McFadden, J. P., Mozer, F. S., Delory, G. T., Peria, W., Chaston, C. C., Temerin, M., Elphic, R., Strangeway, R., Pfaff, R., Cattel, C. A., Klumpar, D., Shelley, E., Peterson, W., Moebius, E., and Kistler, L. : FAST satellite observations of large-amplitude solitary structures, Geophys. Res. Lett., 25, 2041-2044, 1998a.

Ergun, R. E., Carlson, C. W., Fadden, J. P., Mozer, F. S., Muschietti, L., Roth, I., and Strangeway, R.: Debye-scale plasma structures associated with magnetic-field-aligned electric fields, Phys. Rev. Lett., 81, 826-829, 1998b.

Franz, J. R., Kintner, P. M., and Pickett, J. S.: POLAR observations of coherent electric field structures, Geophys. Res. Lett., 25, 1277-1280, 1998.

Goldman, M. V., Openheim, M. M., and Newman, D. L.: Nonlinear two-stream instabilities as an explanation for auroral bipolar wave structures, Geophys. Res. Lett., 26, 1821-1824, 1999a.

Goldman, M. V., Openheim, M. M., and Newman, D. L.: Theory of localized bipolar wave-structures and nonthermal particle distributions in the auroral ionosphere, Nonlinear Proc. Geophys., 6, 221-228, 1999b.

Gurnett, D. A., Persoon, A. M., Randall, R. F., Odem, D. L., Remington, S. L., Averkamp, T. F., Debower, M. M., Hospodarsky, G. B., Huff, R. L., Kirchner, D. L., Mitchell, M. A., Phan, B. T., Phillips, J. R., Schintler, W. J., Sheyko, P., and Tomash, D. R.: The Polar plasma wave instrument, Space Sci. Rev. 71, 597-622, 1995.

Khazanov, G. V., Moore, T. E., Krivorutsky, E. N., Horwitz, J. L., and Liemohn, M. W.: Lower hybrid turbulence and ponderomotive force effects in space plasmas subjected to large-amplitude low-frequency waves, Geophys. Res. Lett., 23, 797-800, 1996.

Khazanov, G. V., Gamayunov, K. V., and Liemohn, M. W.: Alfvén waves as source of lower-hybrid activity in the ring current region, J. Geophys. Res., 105, 5403-5409, 2000.

Kojima, H., Matsumoto, H., Chikuba, S., Horiyama, S., AshourAbdalla, M., and Anderson, R. R.: Geotail waveform observations of broadband/narrowband electrostatic noise in the distant tail, J. Geophys. Res., 102, 14 439-14 455, 1997.

Lakhina, G. S., Tsurutani, B. T., Kojima, H., and Matsumoto, H.: "Broadband" plasma waves in the boundary layers, J. Geophys. Res., 105, 27 791-27 831, 2000.

Lühr, H. and Klöcker, N.: AMPTE-IRM observations of magnetic cavities near the magnetopause, Geophys. Res. Lett., 14, 186189, 1987.

Matsumoto, H., Kojima, H., Miyatake, T., Omura, Y., Okada, M., and Tsutsui, M.: Electrostatic solitary waves (ESW) in the magnetotail-BEN waveforms observed by GEOTAIL, Geophys. Res. Lett., 21, 2915-2918, 1994.

Miyake, T., Omura, Y., Matsumoto, H., and Kojima, H.: Twodimensional computer simulations of electrostatic solitary waves observed by Geotail spacecraft, J. Geophys. Res., 103, $11841-$ $11850,1998$.

Muschietti, L., Ergun, R. E., Roth, I., and Carlson, C. W.: Phasespace electron holes along magnetic field lines, Geophys. Res. Lett., 26, 1093-1096, 1999a.

Muschietti, L., Roth, I., Ergun, R. E., and Carlson, C. W.: Analysis and simulation of BGK electron holes, Nonlinear Proc. Geophys., 6, 211-219, 1999b.
Omura, Y., Kojima, H., and Matsumoto, H.: Computer simulation of electrostatic solitary waves: A nonlinear model of broadband electrostatic noise, Geophys. Res. Lett., 21, 2923-2926, 1994.

Omura, Y., Matsumoto, H., Miyake, T., and Kojima, H.: Electron beam instabilities as generation mechanisms of electrostatic solitary waves in the magnetotail, J. Geophys. Res., 101, 2685-2697, 1996.

Pickett, J. S., Franz, J. R., Scudder, J. D., Menietti, J. D., Gurnett, D. A., Hospodarsky, G. B., Braunger, R. M., Kintner, P. M., and Kurth, W. S.: Plasma waves observed in the cusp turbulent boundary layer: An analysis of high time resolution wave and particle measurements from the Polar spacecraft, J. Geophys. Res., 106 , 19 081-19099, 2001.

Pickett, J. S., Menietti, J. D., Hospodarsky, G. B., Gurnett, D. A., and Stasiewicz, K.: Analysis of the turbulence observed in the outer cusp boundary layer, Adv. Space Res., 30, 2809-2814, 2002.

Russell, C. T., Snare, R. C., Means, J. D., Pierce, D., Dearborn, D., Larson, M., Barr, G., and Le, G.: The CGS/POLAR magnetic field investigation, Space Sci. Rev., 71, 563-582, 1995.

Scudder, J., Hunsacker, F., Miller, G., Lobell, J., Zawistowski, T., Ogilvie, K., Keller, J., Chornay, D., Herrero, F., Fitzenreiter, R., Fairfield, D., Needall, J., Bodet, D., Googins, J., Kletzing, C., Torbert, R., Vandiver, J., Bentley, R., Fillius, W., McIlwain, C., Whipple, E., and Korth, A.: Hydra A 3-dimensional electron and ion hot plasma instrument for the Polar spacecraft of the CGS mission, Space Sci. Rev. 71, 459-495, 1995.

Singh, N., Loo, S. M., Wells, B. E., and Lakhina, G. S.: Evolution of electron beam generated waves resulting in transverse ion heating and filamentation of the plasma, J. Geophys. Res., 106, 21 165-21 182, 2001a.

Singh, N., Loo, S. M., and Wells, B. E. : Electron hole structure and its stability depending on plasma magnetization, J. Geophys. Res., 106, 21 183-21 198, 2001b.

Stasiewicz, K., Seyler, C. E., Mozer, F. S., Gustafsson, G., Pickett, J. S., and Popielawska, B.: Magnetic bubbles and kinetic Alfvn waves in the high-latitude magnetopause boundary, J. Geophys. Res., 106, 29503-29 514, 2001.

Sugiura, M., Skillman, T. L., Ledley, B., and Heppner, J. P.: "Holes" in the magnetic field near the magnetopause, EOS Trans. AGU., 50, 278, 1969

Treumann, R. A., Bostorm, L., LaBelle, J., and Sckopke, N.: The plasma wave signature of a "Magnetic Hole" in the vicinity of the magnetopause, J. Geophys. Res., 95, 19 099-19 114, 1990

Tsurutani, B. T., Arballo, J. K., Lakhina, G. S., Ho, C. M., Buti, B., Pickett, J. S., and Gurnett, D. A.: Plasma waves in the dayside polar cap boundary layer: Bipolar and monopolar electric pulses and whistler mode waves, Geophys. Res. Lett., 25, 417-420, 1998.

Tsurutani, B. T., Arballo, J. K., Galvan, C., Zhang, L. D., Zhou, X. Y., Lakhina, G. S., Hada, G. T., Pickett, J. S., and Gurnett, D. A. : Polar cap boundary layer waves: An auroral zone phenomena, J. Geophys. Res., 106, 19035-19 056, 2001.

Tsurutani, B. T., Dasgupta, B., Arballo, J. K., Lakhina, G. S., and Pickett, J. S.: Magnetic field turbulence, electron heating, magnetic holes, proton cyclotron waves, and the onset of bipolar pulsess (electron hole) events: a possible unifying scenario, Nonlinear Proc. Geophysics, 21, 27-35, 2003. 